\title{
Histological surprise: callosal tuberculoma presenting as malignant glioma
}

\author{
Farzin Fath-Ordoubadi, Russell J M Lane, Peter G Richards
}

\begin{abstract}
A tumour which on CT is clearly intrinsic, irregular, exhibits patchy enhancement with contrast, and invades periventricular tissues, especially the corpus callosum, is very likely to be a glioma and may not be biopsied. A case is presented with these radiological features in which the tumour proved to be a tuberculoma, with complete clinical and radiological resolution after antituberculous chemotherapy.
\end{abstract}

(F Neurol Neurosurg Psychiatry 1997;63:98-99)

Keywords: corpus callosum; tumour; tuberculoma; cerebral biopsy

Certain CT characteristics are said to be diagnostic of malignant glioma-in particular, involvement of the corpus callosum. ${ }^{12}$ It has been argued that in such patients, histological confirmation by brain biopsy is unnecessary as this could add to the short term morbidity and mortality. ${ }^{2-4}$ We report a patient who presented with clinical features and CT characteristics which were considered diagnostic of malignant glioma, but who proved to have a tuberculoma on histological examination.

\section{Case report}

A middle aged Indian man presented with acute right sided facial and limb weakness from which he initially made a slow recovery. Two weeks later there was a further sudden deterioration with increasing weakness and dysphasia. He was thought to have had a stroke and was treated accordingly. However, two weeks later a neurological opinion was sought as his symptoms had progressed and he was complaining of symptoms of general malaise and headache. $\mathrm{He}$ and his family had no history of tuberculosis, and he had lived in the United Kingdom for many years.

On examination he was alert with a pulse of 86 per minute and blood pressure of 140/80 $\mathrm{mm} \mathrm{Hg}$. His chest was clear, and there was no lymphadenopathy and no liver or spleen enlargement. He was slightly drowsy and had an expressive and nominal dysphasia, although his speech was better preserved in his native Indian language than in English. There was a right upper motor neuron facial palsy and a pronounced right hemiparesis (MRC grade 3 ), with a pyramidal distribution. Reflexes were exaggerated on the right, with a Babinski sign, and he exhibited a right sided grasp reflex. A chest radiograph showed no evidence of pulmonary tuberculosis. Contrast enhanced CT was performed (figure A). This was reported as showing a single large, irregular enhancing lesion crossing the midline, considered diagnostic of an extensive malignant glioma arising from the corpus callosum. The patient was treated with dexamethasone.

Despite the radiological findings, and in view of his country of origin, a burr hole brain biopsy was performed. The gross appearance of the biopsy specimen at operation supported the neuroradiological diagnosis of a malignant glioma. However, histological examination showed a necrotising, granulomatous inflammatory lesion and Ziehl-Neilsen staining confirmed the presence of acid fast bacilli. He was treated with $300 \mathrm{mg}$ isonazid, $600 \mathrm{mg}$ rifampacin, and 2 g pyrazinamide daily, and dexamethasone was stopped. Two weeks later at discharge, there was almost complete resolution of dysphasia, and only very mild residual right sided weakness. However, two weeks later he was readmitted, having had several attacks of left sided focal epileptic fits and recurrence of the right hemiparesis and dysphasia. Dexamethasone was recommenced and the dosage of his antituberculous chemotherapy was increased to seven Rifater tablets (each containing $120 \mathrm{mg}$ rifampicin, $50 \mathrm{mg}$ isoniazid, and $300 \mathrm{mg}$ pyrazinamide) daily. In addition he was treated with intramuscular streptomycin for six weeks together with phenytoin, $500 \mathrm{mg}$ daily. He improved steadily and was discharged with minimal weakness and dysphasia, and good control of his seizures. The Rifater was continued until July 1992 (total duration of antituberculous therapy 27 months) and the phenytoin was discontinued a year later. On review in July 1996, he remained asymptomatic and CT showed complete resolution of the lesion (figure $\mathrm{B}$ ).

\section{Discussion}

Malignant glioma still carries an extremely bleak prognosis. ${ }^{4}$ Tumour resection and radiotherapy, although having some palliative benefit, do not seem to greatly influence the 

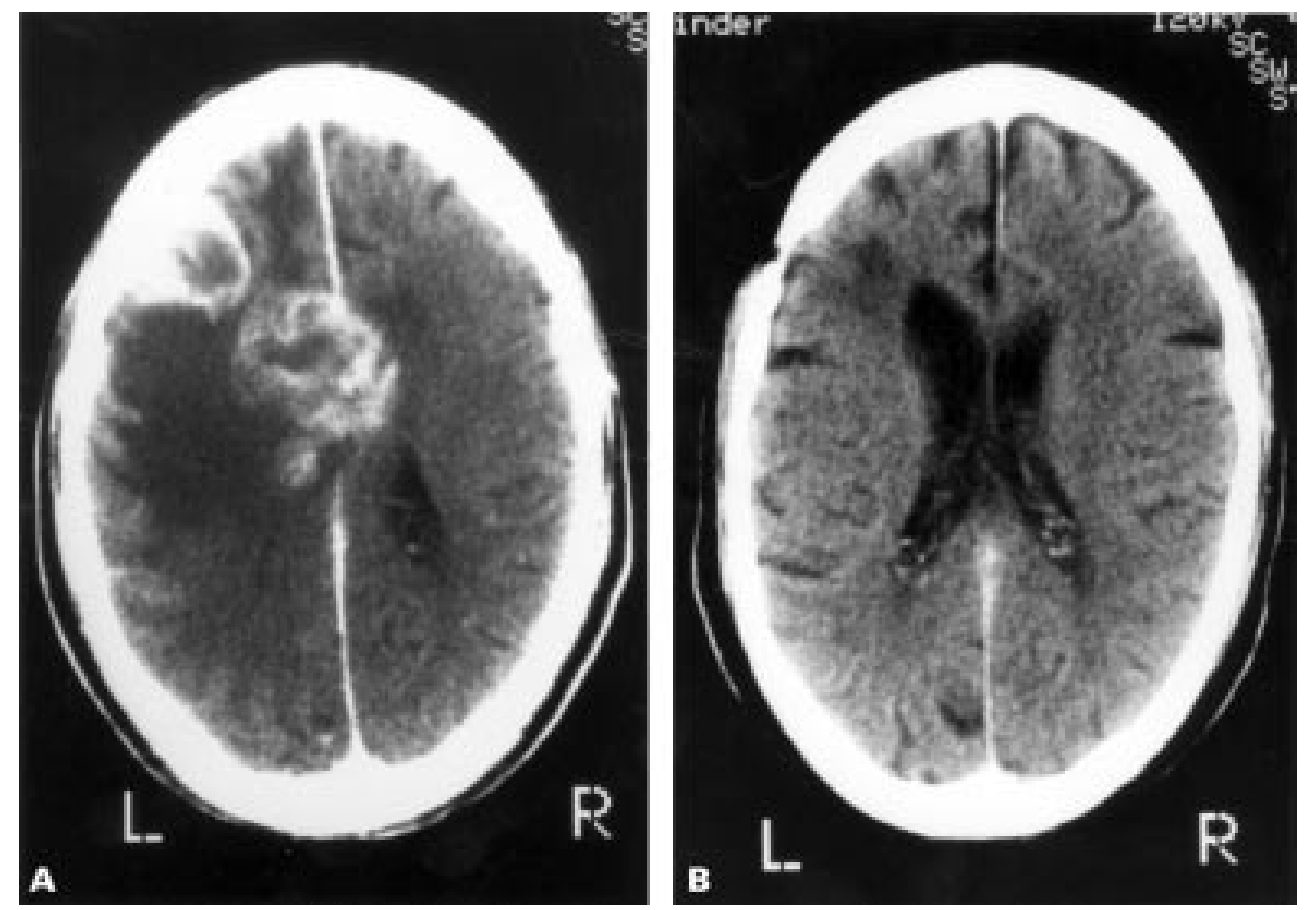

(A) Brain CT at the time of presentation in February 1990. Serial images showed a single large, irregular contrast enhancing lesion arising from the corpus callosum, reported as malignant glioma. (B) Brain CT at the same levels, in fune 1996, showing complete resolution of the lesion.

outcome. ${ }^{3}{ }^{4}$ However, there is disagreement as to whether patients with CT appearances strongly suggestive of a malignant glioma should be subjected to brain biopsy, as this carries a risk of death or significant morbidity of between $1 \%-5 \%$ in various series. ${ }^{45}$

In a retrospective study of 205 patients with glioma, of which 133 had undergone a brain biopsy, Wroe et al found the outcome to be the same in the biopsied and non-biopsied groups, but the short term morbidity was less in those who had not undergone biopsy. They concluded that a conservative approach in such patients was ethical. However, making a firm diagnosis of supratentorial glioma on CT appearances alone in every case is not without risk. ${ }^{6}$ Baker et al reviewed 3000 scans and found 13 out of $464(2.8 \%)$ non-malignant lesions diagnosed as gliomas. In the study of Wroe et $a l^{\beta} 6 / 133$ (4.5\%) patients considered to have gliomas on CT appearances proved to have other diseases on biopsy, including benign lesions in three cases. In a later study, ${ }^{2}$ three neuroradiologists reviewed the scans of 300 patients who had supratentorial mass lesions on CT and who subsequently had histological confirmation of the lesion. The overall error rate was $4.5 \%(12 / 254)$; nine of these errors were benign conditions misdiagnosed as malignant. However, among these patients, a subgroup was identified with CT appearances so specific for malignant glioma that a biopsy was claimed to be unnecessary. In this subgroup, which accounted for $1 / 5$ th of the malignant glioma cases studied, the tumour was "clearly intrinsic, irregular, exhibited patchy enhancement, and invaded the periventricular tissues especially the corpus callosum". When these criteria were present the neuroradiologist made the correct diagnosis of malignant glioma every time. The CT of our patient exhibited all of these criteria.

We conclude that tuberculoma can present clinically and have a CT appearance of a type said to be highly specific for malignant glioma. The only way to distinguish between these two lesions is by histological confirmation by biopsy, or alternatively by treating the patient with a full course of antituberculous drugs and rescanning after three months, in which case a tuberculoma should show evidence of regression. ${ }^{8}$

We thank Dr Leslie Bridges for reporting the pathological findings in this case.

1 McNeil B J, Hanley JA, Funkenstein HH, Wallman J. Paired receiver operating characteristic curves and the effect of receiver operating characteristic curves and the effect of
history on radiographic interpretation. Radiology history on $1982 ; 149: 75-7$.

2 Choksey MS, Valentine A, Shawdon H, Freer CEL, Lindsay $\mathrm{KW}$. Computed tomography in the diagnosis of malignant brain tumours: do all patients require biopsy? $\mathcal{F}$ Neurol Neurosurg Psychiatry 1989;52:821-5.

3 Wroe SJ, Foy, Shaw MDM, Williams IR, Chadwick, West C, Towns G. Differences between neurological and neurosurgical approaches in the management of malignant brain tumours. BMF 1986;293:1015-8.

4 Whittle IR. Management of primary malignant brain tumours. F Neurol Neurosurg Psychiatry 1996; 60: 2-5.

5 DGT Thomas, Nouby RM. Experience in 300 cases of CT-directed stereotactic surgery for lesion biopsy and aspiration of haematoma. Br $\mathcal{F}$ Neurosurg 1989;3:321-6.

6 Kendall BE, Jakubowski J, Pullicino P, Symon L. Difficulties in the diagnosis of supratentorial gliomas by CAT scan. $\mathcal{F}$ in the diagnosis of supratentorial gliomas by

7 Baker HL, Houser OW, Campbell JK. National Cancer Institute Study: evaluation of computed tomography in the diagnosis of intracranial neoplasms. Radiology 1980;136: 91-6.

8 Choudhury AR. Non-surgical treatment of tuberculomas of the brain. Br F Neurosurg 1989;3:643-53. 